\title{
URETEROLITÍASE BILATERAL ASSOCIADA A DOENÇA RENAL CRÔNICA EM \\ FELINO - RELATO DE CASO
}

\author{
PEREIRA, Marcy Lancia ${ }^{1}$; \\ POPP, Priscila ${ }^{2}$ \\ CÉSAR, Jane Regina França ${ }^{3}$.
}

Recebido: $27 / 03 / 2019$

Aceito: $21 / 11 / 2019$

${ }^{1}$ Médica Veterinária, Doutora, Professora, Universidade Federal de Santa Catarina - Curitibanos;

${ }^{2}$ Médica Veterinária autônoma; ${ }^{3}$ Médica Veterinária, Mestre, Centro de Diagnóstico e Especialidades Veterinárias - CDEV, Campinas-SP.

\section{RESUMO}

reterolitíases são importantes causas de obstrução do trato urinário superior em gatos. A ocorrência de cálculos em ambos os ureteres é pouco frequente em felinos. Este relato visa apresentar o caso de uma gata, sem raça definida, com aproximadamente sete anos de idade, com diagnóstico de ureterolitíase e hidronefrose bilaterais, associadas a doença renal crônica. A paciente foi encaminhada devido à queixa de vômito e anorexia. Os cálculos bilaterais em região proximal ureteral foram observados por meio de ultrassonografia e radiografia simples, enquanto hidronefrose e diminuição da relação corticomedular e hiperecogenicidade de cortical foram vistos à ultrassonografia. Inicialmente, foi realizado tratamento clínico com fluidoterapia para manutenção e reposição e analgésico por opção do tutor. Devido à piora do quadro clínico, optou-se por tratamento cirúrgico, entretanto, devido a aspecto macroscópico bastante desfavorável dos rins à celiotomia, optou-se pela eutanásia. No exame post mortem imediato, notou-se dilatação de pelve e cálices renais, atrofia do parênquima renal, irregularidade de contornos renais e presença de urólitos acastanhados em formato de roseta, sugestivos de oxalato de cálcio, em ambos os ureteres. Destaca-se a importância da avaliação por imagem como ferramenta diagnóstica precoce de forma a otimizar o prognóstico para o paciente.

Palavras-chave: Obstrução. Hidronefrose. Gato. Hipercalciúria. Oxalato de cálcio. 


\section{INTRODUÇÃO}

Ureterolitíases são importantes causas de obstrução do trato urinário superior em gatos (PALM; WESTROPP, 2011). Os cálculos oriundos dos rins movem-se por meio de peristaltismo e fluxo passivo de urina até alojarem-se em um ou ambos os ureteres. $\mathrm{Na}$ espécie felina, o aumento da ocorrência das ureterolitíases, nos últimos 20 anos, acompanhou o crescimento da incidência de oxalato de cálcio (FISCHER, 2006). Ainda, a particularidade anatômica dos ureteres felinos justifica a maior ocorrência de ureterolitíase em gatos quando comparada aos cães, uma vez que o lúmen ureteral felino tem aproximadamente 0,4 mm de diâmetro (KOCHIN et al., 1993), bem menos calibroso do que o canino, que tem de 1,3 a 2,7 mm (ROZEAR; TIDWELL, 2003).

Cerca de 2 a 3\% dos cálculos encaminhados a centros de análises são advindos do trato urinário superior em gatos (PIMENTA et al., 2014). Kyles et al. (2005a), em um estudo retrospectivo que envolveu 163 felinos com cálculos ureterais, demonstraram que a utilização de radiografia abdominal e ultrassonografia, juntas ou isoladamente, revelaram cálculos ureterais em 95\% dos gatos, sendo 76\% unilaterais e 19\% bilaterais.

A hidronefrose é caracterizada pela dilatação da pelve renal e consequente degeneração devido a obstrução do fluxo urinário em um ou ambos os rins (SOUZA et al., 2015). Como causas, têm-se os cálculos ureterais, estenoses ureterais, coágulos sanguíneos, tampões de tecidos moles, ectopia ureteral, neoplasia ureteral e ligadura iatrogênica da vesícula urinária (HUDSON; HOLLAND, 2011; MARSHALL, 2011).

Doença renal crônica (DRC) pode demorar a se tornar aparente, já que insuficiência ocorre somente quando $75 \%$ dos néfrons encontram-se lesados. Sinais de uremia normalmente são evidenciados quando 80 a $85 \%$ de lesão está presente (GRAUER, 2005). Em felinos com DRC pré-existente, obstrução ureteral parcial ou completa normalmente resulta de descompensação "aguda-em-crônica", podendo ocorrer hipercalemia e/ou acidemia. Ainda, muitos felinos com DRC também apresentam nefrólitos de oxalato de cálcio (GRAUER, 2015).

O objetivo deste trabalho foi relatar o caso de uma paciente felina adulta diagnosticada com hidronefrose bilateral por ureterólitos, uremia e doença renal crônica. 


\section{RELATO DE CASO}

Foi encaminhada para atendimento pelo Serviço de Nefrologia e Urologia do Centro de Diagnóstico e Especialidades Saúde e Ciência Animal (SCAN), Campinas, SP, Brasil, uma gata sem raça definida, castrada, com aproximadamente sete anos e 3,2 kg de peso vivo, com histórico de vômitos e hiporexia há um mês. No momento da consulta, procedeu-se a coleta de material biológico para hemograma, bioquímica sérica e urinálise, cujos resultados foram: anemia normocítica normocrômica, leucocitose por neutrofilia sem desvio à esquerda e monocitose; azotemia (creatinina de $9,2 \mathrm{mg} / \mathrm{dL}$ ) e isostenúria, respectivamente. Cálculos bilaterais $(0,6$ e $0,8 \mathrm{~cm})$ em região proximal ureteral foram observados por meio de ultrassonografia e radiografia simples, enquanto hidronefrose e diminuição da relação corticomedular e hiperecogenicidade de cortical foram vistas à ultrassonografia.

A paciente foi submetida a tratamento clínico com fluidoterapia, para manutenção e reposição da hidratação, e dipirona ( $25 \mathrm{mg} / \mathrm{kg}$ BID SC) por 2 dias. Foi indicado tratamento cirúrgico, porém o tutor optou por aguardar o deslocamento dos cálculos, mediante manejo clínico, de forma a evitar a cirurgia, já que o prognóstico era desfavorável. Não houve melhora clínica, entretanto, observou-se, radiologicamente, deslocamento dos cálculos, equivalente a meia vértebra lombar. Foram prescritos butilescopolamina (0,3 mg BID) e meperidina ( $2 \mathrm{mg} / \mathrm{kg} \mathrm{SC}$ ), havendo melhora subsequente. Três dias após, a paciente apresentou piora do quadro clínico e a creatinina sérica aumentou para $14,5 \mathrm{mg} / \mathrm{dL}$, optando-se, então, pela cirurgia, cuja abordagem seria a ureterotomia associada a passagem de "stent". Entretanto, devido ao aspecto macroscópico bastante desfavorável dos rins (intensa irregularidade de parede e assimetria visível) à celiotomia, optou-se pela eutanásia. No exame post mortem imediato, notou-se dilatação de pelve e cálices renais, atrofia do parênquima renal, irregularidade de contornos renais, e presença de urólitos acastanhados em formato de roseta, compatíveis com oxalato de cálcio, em ambos os ureteres (Figura 1). 

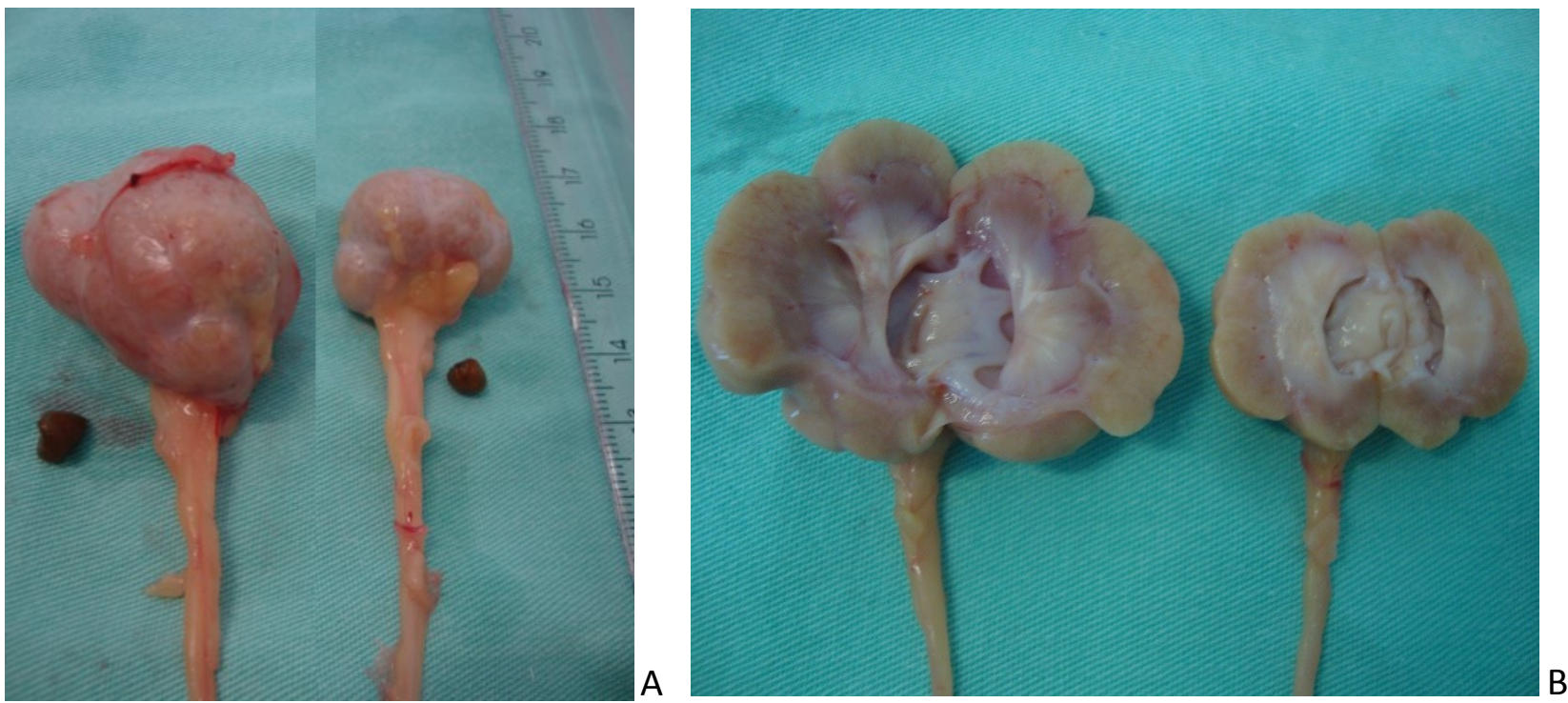

Figura 1 - Imagens post mortem do sistema urinário da paciente felina: Assimetria e irregularidade de parede renal visível antes da ressecção das cápsulas renais, além da visualização dos ureterólitos acastanhados com aspecto de roseta (A) e dilatação de pelve e cálices renais, atrofia do parênquima renal e irregularidade de contornos renais (B).

\section{RESULTADOS E DISCUSSÃO}

No caso relatado, os sinais clínicos iniciaram-se de forma insidiosa e foram piorando ao longo de um mês. Como relatam Palm e Westropp (2011), nas litíases do trato urinário, pode haver inapetência, emese, perda de peso e letargia que, na maioria das vezes, passam despercebidos inicialmente e, com o passar do tempo, o animal acaba se tornando urêmico.

A hipercalciúria representa um fator de risco para formação de cálculos de oxalato de cálcio em gatos. Geralmente é idiopática. Outras causas potenciais de hipercalciúria podem incluir tratamento com diuréticos de alça, corticosteroides, excesso de vitamina C ou $D$, além de dietas com baixos teores de proteínas, fósforo, magnésio, sódio, potássio e umidade (O’KELL et al., 2017). Pelo fato de os cálculos que contêm oxalato de cálcio ( $\mathrm{CaOx}$ ) serem radiopacos e observados frequentemente à radiografia simples abdominal (PALM; WESTROPP, 2011), foi possível a visualização para esta paciente. A análise do cálculo após sua retirada é de grande importância para o manejo clínico do paciente, visto que este tipo de mineral não é dissolvido por meio de tratamento médico conservador (KYLES et al., 2005b). Macroscopicamente, os urólitos de $\mathrm{CaOx}$ são duros, de coloração marrom a preta, podendo ser lisos ou ásperos, além de possuir saliências em forma de amora ou roseta (HESSE; 
NEIGER, 2009). Apesar dos urólitos do presente caso não terem sido enviados para análise, as características radiográficas e macroscópicas foram suficientes para sugerir que se tratavam de $\mathrm{CaOx}$.

O diagnóstico de cálculos ureterais pode ser obtido por meio da radiografia ou ultrassonografia, porém atualmente há um grande desafio nessas modalidades diagnósticas devido a precisão moderada, resultando em falsos-negativos e falsos-positivos. Em virtude disso, a pielografia anterógrada é considerada o padrão ouro para casos de obstruções ureterais (LAMB et al., 2018). É possível, ainda, associar outros meios diagnósticos, como tomografia computadorizada ou ressonância magnética (MACPHAIL, 2015). Neste caso, o quadro clínico associado à azotemia e à ultrassonografia e radiografia simples abdominais permitiram o diagnóstico da ureterolitíase.

O tratamento clínico inicial em casos de ureterolitíases consiste em analgésicos e administração parenteral de fluídos associados a reavaliação periódica. O objetivo do tratamento é promover o deslocamento do cálculo até a vesícula urinária e fornecer suporte para a doença renal, quando instalada (KYLES et al., 2005b; LULICH et al., 2011). Mesmo havendo possibilidade de dilatação de lúmen ureteral correspondente a até 17 vezes do diâmetro original, em resposta ao aumento da diurese (MCLAUGHLIN; BJORLING, 2003), neste caso, o deslocamento dos urólitos foi mínimo em resposta ao tratamento clínico inicial, ou seja, não foi efetivo. Assim, a intervenção cirúrgica se fazia necessária.

As opções cirúrgicas incluem ureteterotomia, ureterocistotomia, colocação de stent ureteral ou mesmo o uso de bypass ureteral subcutâneo (NESSER et al., 2018). Outros métodos tradicionais incluem ressecção e anastomose ureteral, reimplante ureteral e ureteronefrectomia. Ainda, o enxerto intestinal e o uso de ureteroscopia foram descritos recentemente como opções (CLARKE, 2018).

Na Doença Renal Crônica (DRC), ocorre perda significativa do número de néfrons em decorrência de lesão persistente nos rins, os quais não conseguem manter funções adequadas de homeostase no organismo (CHEW et al., 2011; POLZIN, 2011). Uma lesão renal aguda, única ou repetida, causada por obstrução urinária, nefrotoxinas e pielonefrite, pode levar a DRC (FINCH et al., 2016). Ureterolitíase pode ser tanto causa como 
consequência de DRC e há um crescente número de felinos domésticos apresentando-as concomitantemente (PIMENTA et al., 2014). Neste caso não se estabeleceu qual das enfermidades ocorreu primeiramente, se a DRC, que devido hipercalciúria, não mensurada neste caso, ocasionou a formação das litíases ou se as obstruções ureterais acarretaram DRC, mas pelo fato da hidronefrose não ser tão acentuada, poder-se-ia aventar a primeira opção.

\section{CONCLUSÃO}

Ureterolitíase pode ser comum e importante fator de progressão em gatos com doença renal, porém quando observada em ambos os ureteres, achado pouco comum na rotina, indica prognóstico reservado a ruim. O acompanhamento do paciente através de técnicas por imagem favorece diagnóstico e intervenção precoces, direcionando ao tratamento adequado.

\section{BILATERAL URETEROLITHIASIS ASSOCIATED WITH CHRONIC KIDNEY DISEASE IN FELINE PATIENT - CASE REPORT}

\section{ABSTRACT}

U reterolithiasis are important causes of upper urinary tract obstruction in cats. The occurrence of stones in both ureters is infrequent in felines. This report aims to present the case of a non-defined cat with approximately 7 years, diagnosed with bilateral ureterolithiasis and hydronephrosis associated with chronic kidney disease. The patient was referred due to the complaint of vomiting and anorexia. Bilateral uroliths in the proximal ureteral region were observed by ultrasonography and plain radiography, while hydronephrosis and decreased corticomedullary and cortical hyperechogenicity were seen on ultrasonography. By owner's choice, only clinical treatment with fluid therapy was performed for maintenance and replacement and analgesics. Due to worsening of the clinical picture, surgical treatment attempt was made, however, due to the grossly unfavorable macroscopic aspect of the kidneys at celiotomy, euthanasia was chosen. On immediate post mortem examination, pelvic dilation and renal calyces, renal parenchyma atrophy, irregularity of renal contours, and rosette-shaped uroliths suggestive of calcium oxalate were noted in both ureters. The importance of image evaluation as an early diagnostic tool is emphasized in order to optimize the prognosis for the patient.

Keywords: Obstruction. Hydronephrosis. Cat. Hypercalciuria. Calcium oxalate. 


\section{URETEROLITIASIS BILATERAL ASOCIADA CON LA ENFERMEDAD RENAL CRÓNICA EN FELINO - RELATO DE CASO}

\section{RESUMEN}

U

reterolitiasis son importantes causas de obstrucción del tracto urinario superior en gatos. La ocurrencia de cálculos en ambos uréteres es poco frecuente en felinos. Este relato tiene por objeto presentar el caso de una gata sin raza definida, con aproximadamente 7 años, con diagnóstico de ureterolitosis e hidronefrosis bilaterales, asociadas a enfermedad renal crónica. La paciente fue encaminada debido a la queja de vómito y anorexia. Los cálculos bilaterales en región proximal ureteral fueron observados por ultrasonografía y radiografía simple, mientras que la hidronefrosis y la disminución de la relación corticomedular e hiperecogenicidad de cortical se vieron a la ultrasonografía. Inicialmente, se realizó tratamiento clínico con fluidoterapia para mantenimiento y reposición y analgésico por opción del tutor. Debido al empeoramiento del cuadro clínico, se optó por tratamiento quirúrgico, sin embargo, debido al aspecto macroscópico bastante desfavorable de los riñones a la celiotomía, se optó por la eutanasia. En el examen post mortem inmediato, se notó dilatación de pelvis y cálices renales, atrofia del parénquima renal, irregularidad de contornos renales, y presencia de urólitos pardos en forma de roseta, sugerente de oxalato de calcio, en ambos uréteres. Se destaca la importancia de la evaluación por imagen como herramienta diagnóstica precoz para optimizar el pronóstico para el paciente.

Palabras clave: Obstrucción. Hidronefrosis. Gato. Hipercalciuria. Oxalato de calcio.

\section{REFERÊNCIAS}

CHEW, D. J.; DIBARTOLA, S. P.; SCHENCK, P. Canine and Feline Nephrology and Urology. 2. ed. Missouri: Elsevier Saunders, 2011. 526p.

CLARKE, D. L. Feline ureteral obstructions Part 2: surgical management. Journal of Small Animal Practice, v. 59, n. 7, p. 385-397, 2018.

FINCH, N. C.; SYME, H. M.; ELLIOTT, J. Risk factors for development of chronic kidney disease in cats. Journal of Veterinary Internal Medicine, v. 30, p. 602-610, 2016.

FISCHER, J. R. Acute Ureteral Obstruction in Cats: Diagnosis, Management Options, and Outcomes. Advances in Small Animal Medicine and Surgery, v. 19, n. 7, p. 1-3, 2006.

GRAUER, G. F. Early detection of renal damage and disease in dogs and cats. Veterinary Clinics of North America: Small Animal Practice, v. 35, n. 3, p. 581-596, 2005. 
GRAUER, G. F. Feline Struvite and Calcium Oxalate urolithiasis. TVP - Today's Veterinary Practice, September/October, p. 14-20, 2015. Disponível em:

$<$ https://todaysveterinarypractice.com/feline-struvite-calcium-oxalate-urolithiasis/> .

HESSER, A.; NEIGER, R. Urinary Stones in Small Animal Medicine. Boca Raton: Taylor \& Francis Group, 2009. 176p.

HUDSON, J.; HOLLAND, M. Imaging: The Abdomen. In: NORSWORTHY, G. D.; GRACE, S. F.; CRYSTAL, M. A.; et al. The Feline Patient. lowa: Blackwell, 2011. Cap. 292, p. 784-827.

KOCHIN, E. J.; GREGORY, C. R.; WISNER, E.; et al. Evaluation of a method of ureteroneocystostomy in cats. Journal of the American Veterinary Medical Association, $\mathrm{v}$. 202, n. 2, p. 257-260, 1993.

KYLES, A. E.; HARDIE, E. M.; WOODEN, B. G.; et al. Clinical, clinicopathological, radiographic and ultrasonographig abdormalities in cats with ureteral calculi: 163 cases (1984-2002). Journal of the American Veterinary Medical Association, v. 226, n. 6, p. 932-936, 2005a.

KYLES, A. E.; HARDIE E. M.; WOODEN, B. G.; et al. Management and outcome of cats with ureteral calculi: 153 cases (1984-2002). Journal of The American Veterinary Medical Association, v. 226, n. 6, p. 937-944, 2005b.

LAMB, C. R.; CORTELLINI, S.; HALFACREE, Z. Ultrasonography in the diagnosis and management of cats with ureteral obstruction. Journal of Feline Medicine and Surgery, v. 20, n. 1, p. 15-22, 2018.

LULICH, J. P.; OSBORNE, C. A.; ALBASAN, H. Canine and Feline urolithiasis: diagnosis, treatment, and prevention. In: BARTGES, J; POLZIN, D. J. Nephrology and Urology of Small Animals. lowa: Blackwell, 2011. P. 687-706.

MACPHAIL, C. M. Cirurgia do Rim e do Ureter. In: FOSSUM, T. W. Cirurgia de Pequenos Animais. Rio de Janeiro: Elsevier, 2015. Cap. 25, p. 705-734.

MARSHALL, R. Ureteral Obstruction. In: NORSWORTHY, G. D.; GRACE, S. F.; CRYSTAL, M. A.; et al. The Feline Patient. lowa: Blackwell, 2011. Cap. 219, p. 526-529.

MCLAUGHLIN, M. A.; BJORLING, D. E. Ureters. In: SLATTER, D. Textbook of Small Animal Surgery. Philadelphia: Saunders, 2003. Cap. 110, p. 1619-1628.

NESSER, V. E.; REETZ, J. A.; CLARKE, D. L.; et al. Radiographic distribution of ureteral stones in 78 cats. Veterinary Surgery, v. 47, n. 7, p. 895-901, jun. 2018.

O'KELL, A. L.; GRANT, D. C.; KHAN, S. R. Pathogenesis of calcium oxalate urinary stone disease: species comparison of humans, dogs, and cats. Urolithiasis, v. 45, n. 4, p. 329-336, 2017. 
PALM, C. A.; WESTROPP, J. L. Cats and calcium oxalate: Strategies for managing lower and upper tract stone disease. Journal of Feline Medicine and Surgery, v. 13, n. 9, p. 651-660, 2011.

PIMENTA, M. M.; RECHE-JÚNIOR, A.; FREITAS, M. F.; et al. Estudo da ocorrência de litíase renal e ureteral em gatos com doença renal crônica. Pesquisa Veterinária Brasileira, v. 34, n. 6, p. 555-561, 2014.

POLZIN, D. J. Chronic kidney disease. In: POLZIN, D. J.; BARTGES, J. Nephrology and Urology of Small Animals. lowa: Blackweel, 2011. Cap. 48, p. 433-471.

ROZEAR, L.; TIDWELL, A. S. Evaluation of the ureter and ureterovesicular junction using helical computed tomographic excretory urography in healthy dogs. Veterinary Radiology \& Ultrasound, v. 44, n. 2, p. 155-164, 2003.

SOUZA, R.; ZAGGO, E.; MONTEIRO, R. C. P. Hidronefrose por Obstrução de Ureter em Gato: Relato de Caso. Revista Científica de Medicina Veterinária, v. 13, n. 25, p. 1-6, 2015.

Autor para correspondência: Marcy Lancia Pereira.

Universidade Federal de Santa Catarina, Campus Curitibanos, Rodovia Ulysses Gaboardi, km 3, Curitibanos (SC), caixa postal 101, CEP 89.520-000. marcy.pereira@ufsc.br 